\title{
Hamilton-Jacobi formalism on the Null-Plane: Applications
}

\section{C. Bertin}

Instituto de Física Teórica - São Paulo State University, Rua Dr. Bento Teobaldo Ferraz, 271 Bloco II - Barra Funda, 01140-070, São Paulo, SP, Brazil.

E-mail: mcbertineift.unesp.br

\section{B. M. Pimentel}

Instituto de Física Teórica - São Paulo State University, Rua Dr. Bento Teobaldo Ferraz, 271 Bloco II - Barra Funda, 01140-070, São Paulo, SP, Brazil.

E-mail: pimenteldift.unesp.br

\section{E. Valcárcel*}

Instituto de Física Teórica - São Paulo State University, Rua Dr. Bento Teobaldo Ferraz, 271 Bloco II - Barra Funda, 01140-070, São Paulo, SP, Brazil.

E-mail: valcarceleift.unesp.br

\section{G. E. R. Zambrano ${ }^{\dagger}$}

Instituto de Física Teórica - São Paulo State University, Rua Dr. Bento Teobaldo Ferraz, 271 Bloco II - Barra Funda, 01140-070, São Paulo, SP, Brazil.

E-mail: gramoseift.unesp.br

In this work we discuss the Hamilton-Jacobi formalism for fields on the null-plane. The Real Scalar Field in $(1+1)$ - dimensions is studied since in it lays crucial points that are presented in more structured fields as the Electromagnetic case. The Hamilton-Jacobi formalism leads to the equations of motion for these systems after computing their respective Generalized Brackets.

5th International School on Field Theory and Gravitation,

April 20 - 242009

Cuiabá, Brazil

\footnotetext{
*Poster section

†n leave of absence from Departamento de Física, Universidad de Nariño, San Juan de Pasto, Nariño, Colombia.
} 


\section{Introduction}

A physical system is called singular when the determinant of its Hessian matrix is zero. For these systems we cannot perform the Legendre transformation, and then, the Hamiltonian function is not well-defined. Dirac's constraint formalism [1] is the usual method to analyze singular systems. In this formalism the constrains are divided between first and second-class, the first ones being generators of gauge transformations, in accordance to Dirac's conjecture, and the second ones with which it is possible to build Dirac's Brackets.

Carathéodory showed [2] that, for regular systems, we can use equivalent Lagrangians to obtain the Hamilton-Jacobi (HJ) equation directly from the Least Action Principle. Güler [3] has generalized this procedure for singular system. In this case, if the system has $k$ canonical constraints, it is necessary to consider $k+1 \mathrm{HJ}$ differential equations. In order to have an integrable system we need to fulfill the Frobenius' integrability condition, which has shown to be equivalent to the Consistency Condition of the Dirac Formalism [4].

Since Güler's work, several improvements have been done, from working with second order Lagrangians [5], higher-order Lagrangians [6], first-order Lagrangians [7], higher derivatives [8], and later the construction of Generalized Brackets (GBs) for any non-involutive system [9].

In [10], Dirac showed a new time variable to describe the dynamical evolution of a system. This time variable, $\tau \equiv x^{+}$, is a light-cone coordinate, used to describe the plane of all the light rays in the Minkowski space-time; this is the null-plane. Huszar [11] and Steinhardt [12] have studied systems on the null-plane using the Dirac formalism. The aim of this paper is to show two examples of fields on the null-plane using the HJ formalism. First, we study the Real Scalar Field in $(1+1)$ - dimensions, and then, the Electromagnetic Field in $(1+3)$ - dimensions. For all these fields we build their GBs, and field equations.

\section{The Scalar field in $(1+1)$ - dimensions}

In $(1+d)$ - dimensions, the light-cone coordinates are defined as

$$
x^{+} \equiv \frac{1}{\sqrt{2}}\left(x^{0}+x^{d}\right), \quad x^{-} \equiv \frac{1}{\sqrt{2}}\left(x^{0}-x^{d}\right), \quad x^{i}=x^{i}, \quad i=1, \ldots, d-1 .
$$

At the beginning of the study of constrained fields, we take the Scalar Field in (1+1) - dimensions on the null-plane. The Lagrangian density for this system is

$$
\mathscr{L}=\frac{1}{2} \partial_{\mu} \phi \partial^{\mu} \phi-\frac{1}{2} m^{2} \phi^{2}=\partial_{+} \phi \partial_{-} \phi-\frac{1}{2} m^{2} \phi^{2}
$$

whose Euler-Lagrange (EL) equation is the Klein-Gordon-Fock (KGF) equation

$$
2 \partial_{+} \partial_{-} \phi+m^{2} \phi=0 \text {. }
$$

In the context of $\mathrm{HJ}$ formalism, we have two Hamiltonian functions

$$
\begin{aligned}
H^{\prime \tau} & =\int d x^{-} \mathscr{H}^{\prime \tau} \equiv \int d x^{-}\left[\pi^{\tau}+\mathscr{H}_{0}\right]=0, \\
H^{\prime \phi} & =\int d x^{-} \mathscr{H}^{\prime \phi} \equiv \int d x^{-}\left[\pi-\partial_{-} \phi\right]=0,
\end{aligned}
$$


where $\pi^{\tau}=\partial S / \partial \tau, \pi=\partial S / \partial \phi$ is the conjugate momenta, and $H_{0}$ is the Canonical Hamiltonian

$$
H_{0}=\int d x^{-} \mathscr{H}_{0}=\int d x^{-} \frac{1}{2} m^{2} \phi^{2} .
$$

The fundamental differential can be written with the respective Hamiltonian densities

$$
d F=\int d y^{-}\left[\left\{F(x), \mathscr{H}^{\prime \tau}(y)\right\} d \tau+\left\{F(x), \mathscr{H}^{\prime \phi}(y)\right\} d \phi(y)\right]
$$

Now we have to test the integrability condition for $\mathscr{H}^{\phi}$

$$
d \mathscr{H}^{\prime \phi}=-m^{2} \phi d \tau-2 \partial_{-} d \phi=0 .
$$

No new constraint is found.

We have seen that $\mathscr{H}^{\prime \phi}$ is not in involution, since the PB with itself is non zero, then it is possible to build the GBs. In order to achieve this, we have to consider the $1 \times 1$ matrix

$$
M(x, y)=\left\{\mathscr{H}^{\phi}(x), \mathscr{H}^{\phi}(y)\right\}=-2 \partial_{-}^{x} \delta\left(x^{-}-y^{-}\right),
$$

where the index $x$ indicates that the derivation is given with respect to the $x$ point. This matrix has inverse

$$
M^{-1}(x, y)=-\frac{1}{4} \varepsilon\left(x^{-}-y^{-}\right)+f\left(x^{+}\right) .
$$

where $\varepsilon\left(x^{-}-y^{-}\right)$is the sign function, and $f\left(x^{+}\right)$depends on the boundary condition of the problem. The GBs are defined by

$$
\begin{aligned}
\{F(x), G(y)\}^{*}= & \{F(x), G(y)\} \\
& -\int d z^{-} \int d w^{-}\left\{F(x), \mathscr{H}^{\phi}(z)\right\} M^{-1}(z, w)\left\{\mathscr{H}^{\phi}(w), G(y)\right\},
\end{aligned}
$$

from where we get the following the non-zero GBs

$$
\begin{aligned}
& \{\phi(x), \phi(y)\}^{*}=-\frac{1}{4} \varepsilon\left(x^{-}-y^{-}\right)+f\left(x^{+}\right), \\
& \{\phi(x), \pi(y)\}^{*}=\frac{1}{2} \delta\left(x^{-}-y^{-}\right) \\
& \{\pi(x), \pi(y)\}^{*}=-\frac{1}{2} \partial_{-}^{x} \delta\left(x^{-}-y^{-}\right) .
\end{aligned}
$$

We can see that $f\left(x^{+}\right)$is only present in one of the GBs.

Since $M(x, y)$ is regular, we can build the inverse, and we can represent the evolution of any function $F(x)$ with only one parameter, $\tau$. This means that the equations of motion now are given by

$$
d F=\left\{F(x), H^{\prime \tau}\right\}^{*} d \tau=\int d y^{-}\left\{F(x), \mathscr{H}^{\prime \tau}(y)\right\}^{*} d \tau
$$

Then we have

$$
\begin{aligned}
& \partial_{+} \phi=m^{2} \int d y^{-}\left[-\frac{1}{4} \varepsilon\left(x^{-}-y^{-}\right)+f\left(x^{+}\right)\right] \phi(y), \\
& \partial_{+} \pi=-\frac{1}{2} m^{2} \phi .
\end{aligned}
$$


If we derive (2.16) with respect to $x^{-}$and, since $\partial_{-} f\left(x^{+}\right)=0$, we get the KGF equation in lightcone coordinates (2.3). We observe that the KGF equation is independent of the value of the function $f\left(x^{+}\right)$. However, the uniqueness of the equation of motion $(2.16,2.17)$, and of the GB is guaranteed only if an appropriate boundary condition is imposed on the field.

\section{The Electromagnetic field}

Now we will study the Electromagnetic field in $(1+3)$ - dimensions on the null-plane. The Lagrangian density is given by

$$
\mathscr{L}=-\frac{1}{4} F_{\mu v} F^{\mu v}=-\frac{1}{4}\left[-2 F_{+-}^{2}+4 \eta^{i j} F_{+i} F_{-j}+\eta^{i j} \eta^{k l} F_{i k} F_{j l}\right],
$$

where $F_{\mu v}=\partial_{\mu} A_{v}-\partial_{v} A_{\mu}$. The EL equations in light-cone coordinates are

$$
\begin{aligned}
& 0=\partial_{-} F^{-+}+\partial_{i} F^{i+}, \\
& 0=\partial_{+} F^{+-}+\partial_{i} F^{i-}, \\
& 0=\partial_{+} F^{+i}+\partial_{-} F^{-i}+\partial_{j} F^{j i} .
\end{aligned}
$$

In the HJ formalism, the Electromagnetic field has four Hamiltonian functions

$$
\begin{aligned}
H^{\prime \tau} & =\int d^{3} x \mathscr{H}^{\prime \tau} \equiv \int d^{3} x\left[\pi^{\tau}+\mathscr{H}_{0}\right]=0, \\
H^{\prime+} & =\int d^{3} x \mathscr{H}^{\prime+} \equiv \int d^{3} x \pi^{+}=0, \\
H^{\prime i} & =\int d^{3} x \mathscr{H}^{\prime i} \equiv \int d^{3} x\left[\pi^{i}+\eta^{i j} F_{-j}\right]=0, i=1,2,
\end{aligned}
$$

where we used the notation $d^{3} x \equiv d x^{-} d^{2} x$, and the Canonical Hamiltonian density is given by

$$
\mathscr{H}_{0}=\frac{1}{2}\left(\pi^{-}\right)^{2}+\frac{1}{4} \eta^{i j} \eta^{k l} F_{i k} F_{j l}-A_{+}\left(\partial_{i} \pi^{i}+\partial_{-} \pi^{-}\right) .
$$

Indeed, we have a dynamical relationship

$$
\pi^{-} \equiv \frac{\partial \mathscr{L}}{\partial\left(\partial_{+} A_{-}\right)}=F_{+-} .
$$

The fundamental differential is

$$
d F=\int d^{3} y\left[\left\{F, \mathscr{H}^{\prime \tau}(y)\right\} d \tau+\left\{F, \mathscr{H}^{\prime+}(y)\right\} d A_{+}(y)+\left\{F, \mathscr{H}^{\prime i}(y)\right\} d A_{i}(y)\right]
$$

since $\tau, A_{+}$and $A_{i}$ are the independent variables of this theory. Using (3.10) we set $d \mathscr{H}^{{ }^{+}}=0$, in order to test the integrability condition which gives us a new constraint

$$
H^{\prime \omega}=\int d^{3} x \mathscr{H}^{\prime \omega} \equiv \int d^{3} x\left[\partial_{i} \pi^{i}+\partial_{-} \pi^{-}\right]=0
$$

while the integrability condition for $\mathscr{H}^{\prime i}$, that is, $d \mathscr{H}^{\prime i}=0$, gives us a relation

$$
d \mathscr{H}^{\prime i}=\left(\eta^{i m} \eta^{k l} \partial_{l} F_{k m}-\eta^{i j} \partial_{j} \pi^{-}\right) d \tau+2 \eta^{i j} \partial_{-} d A_{j}=0 .
$$


The integrability condition for $H^{\prime \omega}$ is identically satisfied since $F_{\mu \nu}$ is antisymmetric, and no more Hamiltonian functions are given. The system is, therefore, complete.

The matrix of the Poisson brackets of the Hamiltonians $\mathscr{H}^{\prime+}, \mathscr{H}^{\prime i}$ and $\mathscr{H}^{\prime \omega}$ is singular, and the system is still not integrable, since $\mathscr{H}^{\prime i}$ are non-involutive. The largest regular sub-matrix is given by

$$
M^{i j}(x, y) \equiv\left\{\mathscr{H}^{\prime i}(x), \mathscr{H}^{\prime j}(x)\right\}=2 \eta^{i j} \partial_{-}^{x} \delta\left(x^{-}-y^{-}\right) \delta^{2}(\mathbf{x}-\mathbf{y}),
$$

which is regular and has inverse

$$
M_{i j}^{-1}(x, y)=\frac{1}{4} \eta_{i j} \varepsilon\left(x^{-}-y^{-}\right) \delta^{2}(\mathbf{x}-\mathbf{y}) .
$$

The uniqueness is guaranteed imposing boundary conditions to the gauge field. With this inverse the GBs are defined by

$$
\begin{aligned}
\{F(x), G(y)\}^{*}= & \{F(x), G(y)\} \\
& -\int d^{3} z \int d^{3} w\left\{F(x), \mathscr{H}^{i i}(z)\right\}\left[M_{i j}^{-1}\right](z, w)\left\{\mathscr{H}^{\prime j}(w), G(y)\right\},
\end{aligned}
$$

and the fundamental GBs are $\left(\delta^{3}(x-y) \equiv \delta\left(x^{-}-y^{-}\right) \delta^{2}(\mathbf{x}-\mathbf{y})\right)$

$$
\begin{aligned}
& \left\{A_{\mu}(x), A_{v}(y)\right\}^{*}=\frac{1}{4} \delta_{\mu}^{i} \delta_{v}^{j} \eta_{i j} \varepsilon\left(x^{-}-y^{-}\right) \delta^{2}(\mathbf{x}-\mathbf{y}) \\
& \left\{A_{\mu}(x), \pi^{v}(y)\right\}^{*}=\delta_{\mu}^{v} \delta^{3}(x-y)-\frac{1}{4} \delta_{\mu}^{k}\left[\delta_{k}^{v} \partial_{-}^{x}-\delta_{-}^{v} \partial_{k}^{x}\right]\left[\varepsilon\left(x^{-}-y^{-}\right) \delta^{2}(\mathbf{x}-\mathbf{y})\right] \\
& \left\{\pi^{\mu}(x), \pi^{v}(y)\right\}^{*}=-\frac{1}{4} \eta^{l k}\left[\delta_{k}^{\mu} \partial_{-}^{x}-\delta_{-}^{\mu} \partial_{k}^{x}\right]\left[\delta_{l}^{v} \partial_{-}^{x}-\delta_{-}^{v} \partial_{l}^{x}\right]\left[\varepsilon\left(x^{-}-y^{-}\right) \delta^{2}(\mathbf{x}-\mathbf{y})\right]
\end{aligned}
$$

Then, equation of motions are given by the fundamental differential

$$
\left.d F=\int d^{3} y\left[\left\{F, \mathscr{H}^{\prime \tau}(y)\right\}^{*} d \tau+\left\{F, \mathscr{H}^{\prime+}(y)\right\}^{*} d A_{+}(y)+\left\{F, \mathscr{H}^{\prime \omega}\right\}^{*}(y)\right\} d \omega(y)\right],
$$

from where we get the following equations

$$
\begin{aligned}
\partial_{+} A_{+} & =0 \\
\partial_{+} A_{-} & =\pi^{-}+\partial_{-} A_{+}-\partial_{-} \partial_{+} \omega \\
\partial_{+} A_{i} & =\frac{1}{4} \int d y^{-} d^{2} y\left[\partial_{i}^{x} \pi^{-}(y)-\eta^{j k} \partial_{k}^{x} F_{j i}(y)\right] \varepsilon\left(x^{-}-y^{-}\right) \delta^{2}(\mathbf{x}-\mathbf{y})-\partial_{i} \partial_{+} \omega \\
\partial_{+} \pi^{+} & =\partial_{i} \pi^{i}+\partial_{-} \pi^{-} \\
\partial_{+} \pi^{-} & =-\frac{1}{4} \eta^{i j} \partial_{i}^{x} \partial_{j}^{x} \int d y^{-} d^{2} y \pi^{-}(y) \varepsilon\left(x^{-}-y^{-}\right) \delta^{2}(\mathbf{x}-\mathbf{y}) \\
\partial_{+} \pi^{i} & =\frac{1}{2} \eta^{i j} \partial_{j}^{x} \pi^{-}+\frac{1}{2} \partial_{j} F^{j i}
\end{aligned}
$$

equation (3.21) is equivalent to the dynamical relationship (3.9) if we set $\partial_{-} \partial_{+} \omega=0$. Since $\omega$ is an arbitrary field, it is always possible to choose it to fulfill this requirement. In fact, the best condition would be to set $\partial_{+} \omega=0$, and in this case, all above characteristics equations becomes consistent to the Euler-Lagrange field equations of the system, as well as to the former integrability conditions. 


\section{Final Remarks}

In this work we analyzed, through the HJ formalism, the Real Scalar field and the Electromagnetic field on the null-plane. In these examples we have deal with a matrix which contains the operator $\partial_{-}^{x} \delta(x-y)$ which is a characteristic of the null-plane dynamics. Since the inverse of this matrix is not unique, we have a family of inverses, all of them differ by a boundary term.

It becomes necessary to choose appropriate boundary conditions to achieve a unique solution of the characteristics equations. This can be achieved by making the boundary terms present on the generalized brackets equal to zero, and then, the dynamics on the reduced phase space becomes well defined.

The GBs for the Real Scalar field are the same obtained using the Dirac formalism. However, the GBs are different from the obtained by the Electromagnetic field. This is due to the fact that the null-plane dynamics requires a lesser number of fields to describe the system. In this last application it is left with a set of involutive constraints, that are equivalent to the first-class constraints in Dirac's formalism. It is our intention, in future works, to study the relations between these constraints and gauge invariance under the scope of the HJ formalism.

\section{Acknowledgments}

M. C. Bertin and C. E. Valcárcel were supported by CAPES. B. M. Pimentel was partially supported by CNPq. G. E. R. Zambrano was supported by CNPq.

\section{References}

[1] P. A. M. Dirac, Canad. J. Math. 2, 129 (1950); Canad. J. Math. 3, 1 (1951); Proc. Roy. Soc. A 246, 326 (1958); Lectures on Quantum Mechanics, Yeshiva University, New York, 1964.

[2] C. Carathéodory, Calculus of Variations and Partial Differential Equations of the First Order, Part I, II, Holden Day Inc, NY, 1967.

[3] Y. Güler, Nuovo Cimento B 100, 251 (1987); J. Math. Phys. 30, 785 (1992); Nuovo Cimento B 107, 1398 (1992).

[4] B. M. Pimentel, R. G. Texeira, J. L. Tomazelli, Ann. Phys 267, 75 (1998).

[5] B.M. Pimentel, R.G. Teixeira, Il Nuovo Cimento B111, 841 (1996).

[6] B.M. Pimentel, R.G. Teixeira, Il Nuovo Cimento B113, 805 (1998).

[7] M. C. Bertin, B. M. Pimentel, P. J. Pompeia, Mod. Phys. Lett. A 20, 2873 (2005).

[8] M. C. Bertin, B. M. Pimentel, P. J. Pompeia, Ann. Phys. 323, 527 (2008).

[9] M.C. Bertin, B.M. Pimentel, C.E. Valcárcel, Ann. Phys. 323, 3137 (2008).

[10] P. A. M. Dirac, Rev. Mod. Phys. 21, 392 (1949).

[11] M. Huszar, J. Phys. A: Math. Gen. 9, 1359 (1976).

[12] P. J. Steinhardt, Ann. Phys 128, 425 (1980). 